\title{
Colloids with valence and specific directional bonding
}

\section{Citation}

Wang, Yufeng, Yu Wang, Dana R. Breed, Vinothan N. Manoharan, Lang Feng, Andrew D. Hollingsworth, Marcus Weck, and David J. Pine. 2012. "Colloids with Valence and Specific Directional Bonding." Nature 491 (7422) (October 31): 51-55. doi:10.1038/nature11564.

\section{Published Version}

doi:10.1038/nature11564

\section{Permanent link}

http://nrs.harvard.edu/urn-3:HUL.InstRepos:24902731

\section{Terms of Use}

This article was downloaded from Harvard University's DASH repository, and is made available under the terms and conditions applicable to Other Posted Material, as set forth at http:// nrs.harvard.edu/urn-3:HUL.InstRepos:dash.current.terms-of-use\#LAA

\section{Share Your Story}

The Harvard community has made this article openly available.

Please share how this access benefits you. Submit a story.

Accessibility 


\title{
Colloids with valence and specific directional bonding
}

\author{
Yufeng Wang1, Yu Wang ${ }^{1}$, Dana R. Breed ${ }^{2}$, Vinothan N. Manoharan ${ }^{3,4}$, Lang
}

Feng ${ }^{5}$, Andrew D. Hollingsworth ${ }^{5}$, Marcus Weck ${ }^{1}$, David J. Pine ${ }^{5 *}$

${ }^{1}$ Molecular Design Institute and Department of Chemistry, New York University, New York, New York 10003, USA.

${ }^{2}$ The Dow Chemical Company, 2301 N. Brazosport Boulevard, Freeport, Texas 77541, USA.

${ }^{3}$ School of Engineering and Applied Sciences, Harvard University, Cambridge, Massachusetts 02138, USA.

${ }^{4}$ Department of Physics, Harvard University, Cambridge, Massachusetts 02138, USA.

${ }^{5}$ Center for Soft Matter Research and Department of Physics, New York University, New York, New York 10003, USA.

*To whom correspondence should be addressed. E-mail: marcus.weck@nyu.edu, pine@nyu.edu.

The ability to design and assemble 3-dimensional structures from colloidal particles is limited by the absence of specific directional bonds. As a result, complex or low-coordination structures, common in atomic and molecular systems, are rare in the colloidal domain. Here we demonstrate a general method for creating the colloidal analogues of atoms with valence: colloidal particles with chemically functionalized patches that can form highly directional bonds. These "colloidal atoms" possess all the common symmetries - and some uncommon ones-characteristic of hybridized atomic orbitals, including $s p, s p^{2}, s p^{3}, s p^{3} d, s p^{3} d^{2}$, and $s p^{3} d^{3}$. Functionalizing the patches with DNA with single-stranded sticky ends makes the interactions between patches on different particles programmable, specific, and reversible, thus facilitating the self-assembly of particles into "colloidal molecules," including “molecules" with triangular, tetrahedral, and other bonding symmetries. Because colloidal dynamics are slow, the kinetics of molecule formation can be followed directly by optical microscopy. These new colloidal atoms should enable the assembly of a rich variety of new micro-structured materials. 


\section{Introduction}

The past decade has seen an explosion in the kinds of colloidal particles that can be synthesized $^{1,2}$, with many new shapes, such as cubes ${ }^{3}$, clusters of spheres ${ }^{4-6}$ and dimpled particles ${ }^{7,8}$ reported. Because the self-assembly of these particles is largely controlled by their geometry, only a few relatively simple crystals have been made: face-centered and body-centered cubic crystals and variants ${ }^{9}$. Colloidal alloys increase the diversity of structures ${ }^{10-12}$, but many structures remain difficult or impossible to make. For example, the diamond lattice, predicted more than 20 years ago to have a full 3-dimensional photonic band gap ${ }^{13}$, still cannot be made by colloidal self-assembly because it requires 4-fold coordination. Without directional bonds, such low-coordination states are unstable.

In contrast to colloids, atoms and molecules control their assembly and packing through valence. In molecules like methane $\left(\mathrm{CH}_{4}\right)$, for example, the valence orbitals of the carbon atom adopt $s p^{3}$ hybridization and form four equivalent $\mathrm{C}-\mathrm{H}$ bonds in a tetrahedral arrangement. In the colloidal domain, the kinds of structures that could be made would vastly increase if particles with controlled symmetries and highly directional interactions were available. What is needed are colloids with valence ${ }^{14}$.

One approach is to decorate the surface of colloidal particles with "sticky patches," made, for example, of synthetic organic or biological molecules and assigned to specific locations ${ }^{15-19}$. Bonding between particles occurs through patch-patch interactions, so that in principle the location and functionality of the patches can endow particles with bonding directionality and valence. This approach is conceptually simple, yet challenging to realize. For example, so-called Janus particles with asymmetrically functionalized surfaces can be made, but normally have only a single patch ${ }^{20-22}$. Triblock Janus particles have also been fabricated by glancing-angle deposition and assembled into a kagome lattice, the 2-dimensional analogue of a diamond crystal $^{23}$. However, only two patches are made using this method, and low quantities of particles are obtained. Other strategies have used faceted particles ${ }^{24}$, particles with protrusions ${ }^{25}$ or coordinated patches ${ }^{26,27,28}$, but 3 -dimensional directional bonding and assembly have yet to be demonstrated ${ }^{29}$.

Here we demonstrate the synthesis and assembly of colloidal particles with directional interactions that mimic those of atoms with either monovalent $s$ or $p$ 
orbitals, or multivalent $s p, s p^{2}, s p^{3}, s p^{3} d, s p^{3} d^{2}$, or $s p^{3} d^{3}$ hybridized orbitals. We do so by making particles with various numbers of patches, $n=1-7$ and higher, that adopt spherical, linear, triangular, tetrahedral, trigonal dipyramidal, octahedral, or pentagonal bipyramidal symmetries. The patches are then site-specifically coated with oligonucleotides, enabling a reversible and controllable attraction between patches on different particles. Using these colloidal "atoms", we demonstrate that a vast collection of colloidal molecules and macromolecules are readily accessible through self-assembly schemes analogous to chemical reactions.

\section{Synthesis}

The fabrication of patchy particles, summarized in Figure 1a, starts with cross-linked amidinated polystyrene microspheres, $540 \mathrm{~nm}$ or $850 \mathrm{~nm}$ in diameter ${ }^{30}$. Small clusters of these microspheres are assembled using an emulsion-evaporation method ${ }^{5}$ that produces so-called "minimal-moment" clusters with reproducible symmetries and configurations: spheres, dumbbells, triangles, tetrahedra, triangular dipyramids, octahedra, and pentagonal dipyramids, for clusters of $n=1$ to 7 particles (Fig. 1b).

Patchy particles are formed from the clusters using a two-stage swelling process followed by polymerization ${ }^{31}$. First, a low-molecular weight, water-insoluble organic compound (1-chlorodecane) is introduced into the colloidal clusters that are suspended in water with surfactant (sodium dodecyl sulfate, SDS). Adding a small amount of acetone to the suspension aids in the transport of the 1-chlorodecane into the colloidal clusters. We also introduce an oil-soluble initiator, benzoyl peroxide (BPO) and 1,2-dichloroethane, which dissolves BPO and is miscible with 1-chlorodecane. Subsequent stripping of the acetone and 1,2-dichloroethane from the solution traps the 1-chlorodecane as well as BPO in the polymer particles. The clusters are then swollen by styrene monomer. The 1-chlorodecane introduced earlier acts as an osmotic swelling agent that increases the amount of monomer that can be absorbed by the clusters ${ }^{32}$. Since each cluster of a given number of particles contains the same amount of swelling agent, chemical equilibrium assures that clusters of the same size all swell by the same amount, with the total amount of swelling controlled by the quantity of added monomer. 


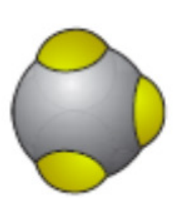

Amidine patches

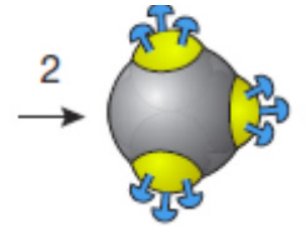

Biotin patches

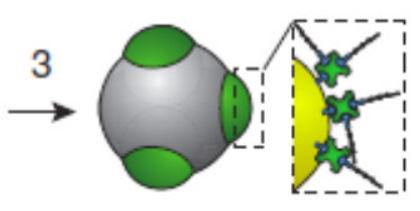

DNA

patches

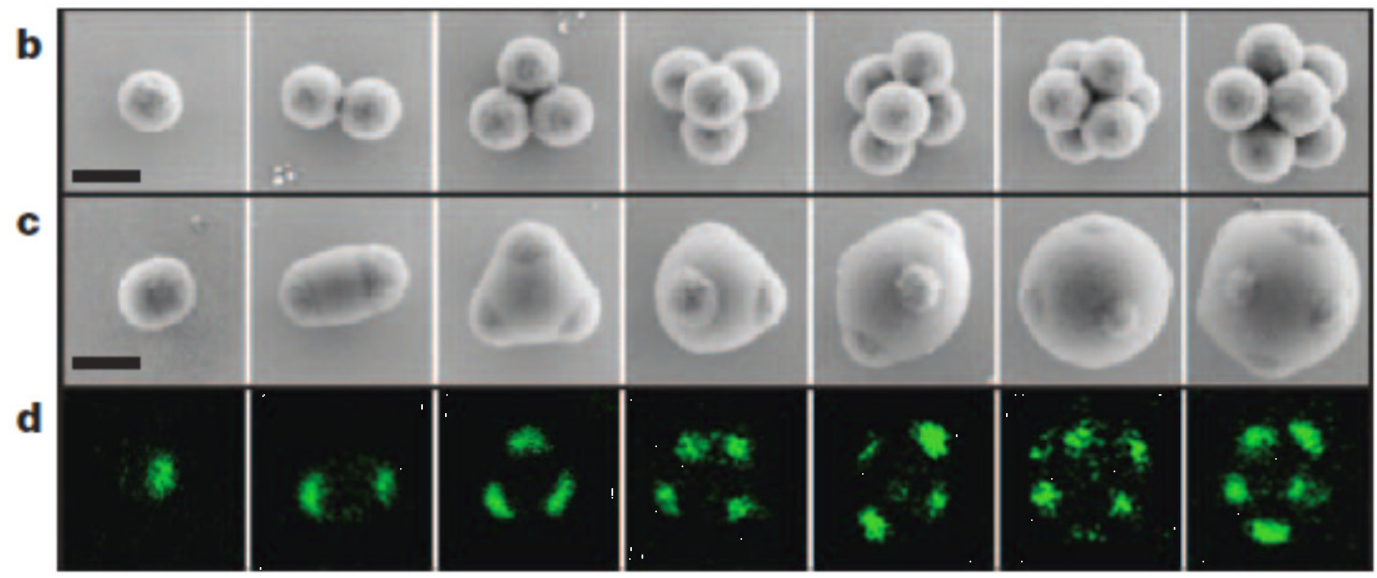

Figure 1 | DNA patchy particle fabrication. a, Scheme illustrating the preparation of colloidal particles with DNA-functionalized patches with well-defined symmetries. A 4-patch particle is shown as an example. (1) A cluster of 4 amidinated polystyrene microspheres, prepared by the method of Manoharan et al., is swollen with styrene such that the extremities of the cluster, a tetrahedron in this case, protrude from the styrene droplet. The styrene is then polymerized and the protrusions from the original cluster become patches. (2) Biotin is site-specifically functionalized on the patches. (3) Biotinated DNA oligomers are introduced and bind to the particle patches via a biotin-streptavidin-biotin linkage. b, Electron micrographs of amidinated colloidal clusters, showing the particle configurations for clusters of $n=1$ to 7 microspheres. c, Electron micrographs of amidinated patchy particles after encapsulation. The patches inherit the symmetries of their parent clusters. d, Confocal fluorescent images verifying the functionalization of DNA oligomers on patchy particles. The fluorescence comes from the dye-labeled streptavidin that links DNA with the patches. Scale bar, $500 \mathrm{~nm}$. 
After swelling, we polymerize the styrene by thermally degrading the BPO previously introduced into each cluster. Swelling is controlled so that the extremities of the original clusters are not encapsulated, but are left as patches. Clusters of the same order $n$ are encapsulated to the same extent, leading to uniform patch configurations, as seen in Figure 1c, which shows SEM images of particles with 1 to 7 patches (see Supplementary Fig. S1a for higher order patches). Using $\mathrm{BPO}$ as the initiator ensures that there are no functional groups introduced, so the surface created by swelling the clusters-the "anti-patch" surface-is chemically inert and different from the patches: only the patches have the functional amidine groups.

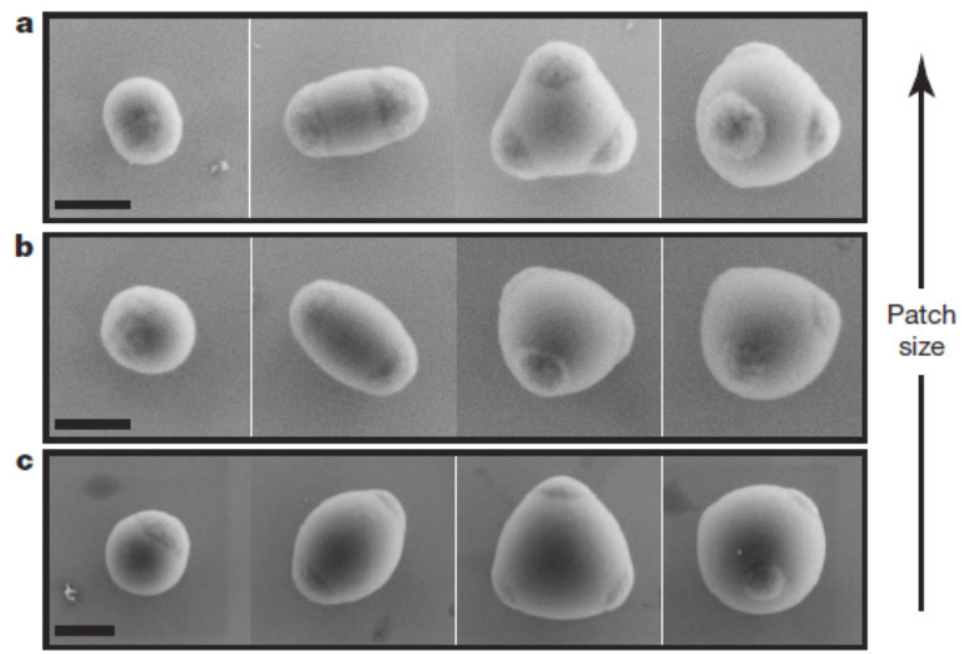

Figure 2 | Control of patch size. Electron micrographs of patchy particles showing that the sizes of patches can be adjusted by changing encapsulation conditions. a, Particles with relatively large patches are fabricated when clusters are swollen with $1.0 \mathrm{~mL}$ of styrene monomer. Primary spheres are $540 \mathrm{~nm}$ in diameter. b, Under identical conditions, smaller patches are obtained when more monomer, $1.2 \mathrm{~mL}$, is added. c, Smaller patches, relative to particle size, are obtained using primary microspheres $850 \mathrm{~nm}$ in diameter. Using larger particles facilitates observation under an optical microscope. Divalent, trivalent and tetravalent particles from this batch are used in the colloidal molecule formation, and the monovalent particles are used in kinetics study, as discussed below. Arrow indicates decreasing patch size. Scale bars, $500 \mathrm{~nm}$.

Patch size is controlled during the swelling process by adjusting the amount of monomer that is introduced: the more monomer that is added, the smaller the patches are. Figure 2 shows that considerable variation in patch size can be 
achieved in this way. Small patches favor greater directionality, while larger patches permit multiple links per patch, as we discuss below.

A key design feature of our method is the use of clusters as intermediates. Their diversity in particle number and symmetry is translated directly to the number and symmetry of the particle patches. In contrast to the planar symmetry of Janus particles $^{23,33}$, the symmetries of these patchy particles are fully 3-dimensional.

\section{Table 1}

\begin{tabular}{cccccccc}
\hline Number of patches & 1 & 2 & 3 & 4 & 5 & 6 & 7 \\
High shear & $61 \%$ & $15 \%$ & $4 \%$ & $1 \%$ & $0.2 \% *$ & $0.02 \% *$ & $0.001 \% *$ \\
Low shear & $7 \%$ & $16 \%$ & $25 \%$ & $15 \%$ & $8 \%$ & $5 \%$ & $3 \%$ \\
\hline
\end{tabular}

Quantities of particles in the different bands. Density gradient centrifugation is used to fractionate the patchy particles. The fraction of identical particles obtained from a single centrifuge tube is shown in the table. Fractions of $10 \%-20 \%$ correspond to $10^{8} \sim 10^{9}$ particles in a single fractionation. *For these higher valence particles, the fractions were estimated from their number ratio relative to lower valence particles observed under a microscope (see Methods).

Our method makes samples containing large scalable quantities of particles having different valence (numbers of patches). Essentially all the starting colloidal particles are converted into particles with one or more patches. Adjusting the emulsification conditions used when making the clusters ${ }^{34}$ changes the relative distribution of particles with different valence. Using a higher shear rate, for example, makes smaller emulsion droplets, which skews the distribution towards lower-valence particles. To fractionate the particles, we use density gradient centrifugation, obtaining up to 12 clear bands corresponding to particles with different valence (see Supplementary Fig. S1b, S1c). Table 1 summarizes the fraction of particles obtained in each band for two different shearing conditions. For the lower shear preparation, each of the four upper bands, which correspond to particles with 1 to 4 patches, contains $10^{8}$ to $10^{9}$ identical particles. For the higher shear preparation, greater quantities are produced in the upper bands and lower quantities are produced in the lower bands. In most cases, we use conditions (see Methods) that produce the most 2-, 3- and 4-patch particles, which are most useful 
for making analogues of common molecules. If pure samples containing patchy particles of identical valence are desired, then it is the fractionation step that ultimately limits the quantity available. Typically, we collect the same valence from up to 40 separations run in parallel, accumulating up to $10^{9}$ to $10^{11}$ particles.

The amidine groups on the colloid surface are crucial to the patchy particle fabrication process. First, the positive charge created from the dissociation of amidine hydrogen chloride salt $\left(-\mathrm{C}(\mathrm{NH}) \mathrm{NH}_{3} \mathrm{Cl}\right)$, along with the SDS surfactant, stabilizes the microspheres as well as the clusters by electrostatic repulsion. Second, when the clusters are swollen and encapsulated, the positive charges make the patches of the cluster more hydrophilic than the monomer-water interface, which is stabilized only by SDS. This difference in interfacial energies leads to finite contact angles and well-defined patches. Most importantly, the amidine groups can be easily functionalized in aqueous solution.

The amidinated patches are functionalized with biotin, to which DNA with single-stranded "sticky" ends is attached using a biotin-streptavidin-biotin linkage. We use sulfo-NHS-biotin (Biotinamidohexanoic acid 3-sulfo-N-hydroxysuccinimide ester sodium salt), a water-soluble biotin derivative, to functionalize the patches. This step is carried out in PBS buffer ( $\mathrm{pH}=7.4)$, where the $\mathrm{N}$-hydroxysuccinimide ester (NHS) can react with amidine groups and covalently link the biotin to the patches.

The DNA oligomer is prepared separately. It has three parts. At the 5' end, it has a biotin as an anchoring molecule. In the middle, there is a 49 base-pair double helix that acts as a spacer. Finally, at the $3^{\prime}$ terminus, a single strand of 11 complementary or 8 palindrome base pairs forms the sticky end (for sequences, see Methods). Mixing DNA with streptavidin in a 1:1 ratio yields a streptavidin-DNA complex, which is then added to the biotin-functionalized patchy particles to produce DNA-functionalized patchy particles. The streptavidin contains a fluorescent tag for visualization by confocal microscopy. Figure $1 \mathrm{~d}$ shows that only the patches of the particle are fluorescent, indicating that the streptavidin-DNA complex successfully coats the particle patches and that the amidine-NHS chemistry used for biotin functionalization works as designed.

The binding between patches on different particles is realized by hybridizing DNA oligomers on different patches. The oligomers, about 18 nanometers in length, provide short-range attractions and thus enforce the directionality defined by the 
particle patches. DNA is widely used for linking nanoparticles because it can be synthesized with control over the length and sequences of the base pairs, which, in turn, controls the specificity and the strength of interaction ${ }^{35-38}$. Hybridization of the complementary strands is fully reversible with temperature so that particle assembly can be controlled by varying temperature.

We functionalize purified patchy particles with complementary DNA strands, designated $\mathrm{R}$ (red) or $\mathrm{G}$ (green), that are designed to bind selectively only to each other, or with a palindrome $\mathrm{P}$ strand, that only binds to other $\mathrm{P}$ strands. To differentiate the particles under the confocal microscope, red fluorescent (Alexa 647) streptavidin is used with $R$ particles, while green fluorescent (Alexa 488) streptavidin is used with G particles (see Supplementary Fig. S2).

\section{Assembly of colloidal molecules}

With our collection of R, G, and P patchy particles, we can build colloidal assemblies that mimic not only the geometry, but also the chemistry of molecules. Figure 3a (left) shows the formation of AB-type colloidal molecules from two 1-patch particles with complementary sticky ends. The system produces colloidal dumbbells without the random aggregation observed using spherical particles uniformly coated with DNA, and consistent with there being only one patch per particle. The confocal fluorescent image in Fig. 3a (middle) shows only complementary R-G particle pairs and no R-R or G-G pairs, confirming that DNA hybridization drives particle assembly. The resulting dumbbells are the colloidal analogues of $\mathrm{AB}$ type molecules such as hydrogen chloride (Fig. 3a, right). Here, in contrast to hydrogen and chlorine, the sizes of the two atoms are the same, although they need not be. Patchy particles of different sizes can be fabricated and DNA bonds of various strengths can be used, so colloidal molecules of different size ratio and bond strength can be obtained.

Figure $3 \mathrm{~b}$ shows linear $\mathrm{AB}_{2}$ type colloidal molecules, the colloidal analogues of molecules like carbon dioxide $\left(\mathrm{CO}_{2}\right)$ or beryllium chloride $\left(\mathrm{BeCl}_{2}\right)$, that are obtained when green divalent (2-patch) particles are mixed with red monovalent particles. Triangle-like $\mathrm{AB}_{3}$ (Fig. 3c) and tetrahedron-like $\mathrm{AB}_{4}$ (Fig. 3d) colloidal molecules are similarly obtained by mixing trivalent (3-patch) particles and tetravalent (4-patch) particles, respectively, with monovalent particles (see Supplementary Movie 1 for all colloidal molecules). 

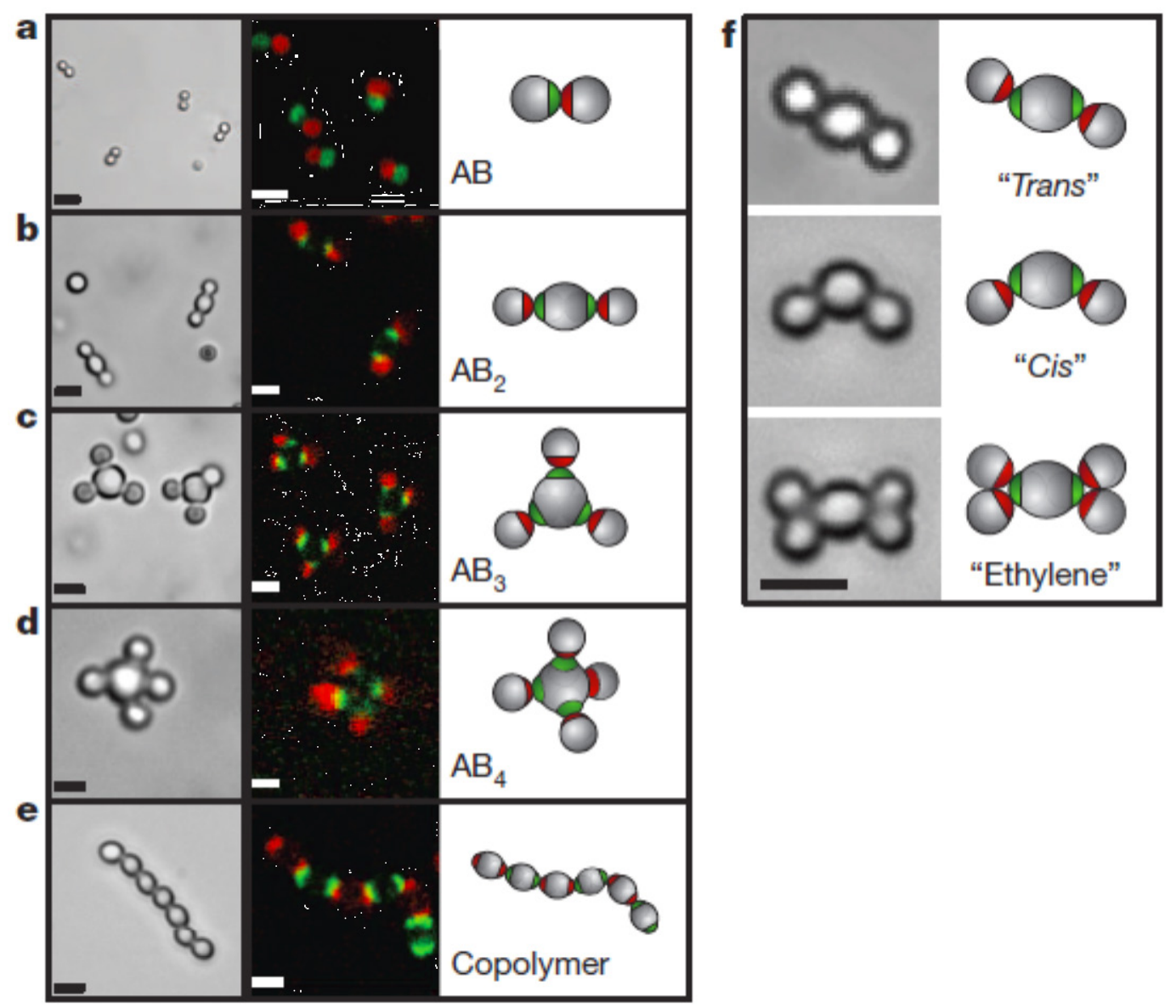

Figure 3 | Specific directional bonding between colloidal atoms observed with optical microscopes. Bright field (left), confocal fluorescent (middle), and schematic images (right), show colloidal molecules self-assembled from patchy particles. a, Complementary green and red monovalent particles form dumbbell-shaped $\mathrm{AB}$ type molecules. Supra-colloidal molecules $\mathrm{AB}_{2}, \mathrm{AB}_{3}$, and $\mathrm{AB}_{4}$ are formed by mixing red monovalent with green (b) divalent, (c) trivalent, and (d) tetravalent particles. e, Mixing complementary divalent particles, linear alternating polymer chain spontaneously assembles. f, When particles with bigger patches are used, cis-trans-like isomers can form. Introducing more monovalent particles leads to ethylene-like colloidal molecules. Scale bars, 2 $\mu \mathrm{m}$.

Bonding specificity is critical for the formation of all of the $A_{n}$ structures. It promotes the formation of $\mathrm{AB}$ bonds while prohibiting the formation of $\mathrm{AA}$ and $\mathrm{BB}$ bonds, ensuring that the trivalent, and tetravalent particles can act as the central atoms, thus mimicking atomic orbitals in geometry and valence. Complementary 
monovalent particles then serve as ligands that form several bonds with the central atom. The confocal images in Fig. 3 (middle) show the directionality and specificity of the interactions between the central atoms and their monovalent particle ligands.

Other structures that can be made include colloidal analogues of alternating copolymers (Fig. 3e), formed using complementary divalent particles. Figure 3e shows that only green and red divalent particles bind to each other.

If particles have patches big enough to accommodate more than one complementary particle, molecular isomers and branched polymers are obtained. Figure $3 \mathrm{f}$ shows two isomers of a non-linear $\mathrm{AB}_{2}$ type that mimic the cis- and transconformations of a double bond. Such isomers may behave quite differently from one another in diffusion, rotation and reactivity. Additional monovalent particles can bind to the isomers and form ethylene-like structures (Fig. 3f, bottom). In the assembly of colloidal polymers from divalent particles, particles with bigger patches lead to branched chains and cross-linked networks (see Supplementary Fig. S3a). These results highlight the importance of controlling the patch size and in particular the ability to make patches sufficiently small such that steric hindrance prevents more than one particle from attaching (see Supplementary Fig. S4 for SEM pictures of patchy particles used in colloidal molecules and geometry analysis for hindrance).

Self-complementary palindrome strands can also be used for self-assembly of mono- and divalent particles. Monovalent particles with palindrome sticky DNA yield $\mathrm{A}_{2}$ type colloidal molecules, analogous to $\mathrm{H}_{2}$ or $\mathrm{Cl}_{2}$, while divalent particles yield homopolymers. One can also envision higher order palindrome particles that might assemble into extended open structures like a diamond lattice ${ }^{39}$.

\section{Colloidal reactions}

The self-assembly of patchy particles into a target structure can be viewed as a "colloidal reaction" or more generally as "supracolloidal chemistry" 40 . As in conventional chemical reactions, colloidal particles with a particular morphology and binding capacity are used as reagents and mixed together stoichiometrically. For example, 4 equivalents of monovalent and 1 equivalent of complementary tetravalent particles assemble into $\mathrm{AB}_{4}$ colloidal molecules. The yield is about $50 \%$ after a few days; that is, $50 \%$ percent of the tetravalent particles have 4 monovalent particles attached, with the remainder consisting of incomplete 
structures like $A B_{3}, A B_{2}$ and $A B$. Using an excess of the monovalent particles increases the yield of the final $\mathrm{AB}_{4}$ product, just as for conventional chemical reactions. A 5-fold excess of monovalent particles, for example, increases the yield of $\mathrm{AB}_{4}$ to $80 \%$ (see Supplementary Fig. S3b). It should also be possible to increase the yield by increasing the strength of the DNA-mediated patch binding, which can be done by increasing the length of the DNA sticky ends or by increasing the density of the DNA attached to the patches ${ }^{41}$.

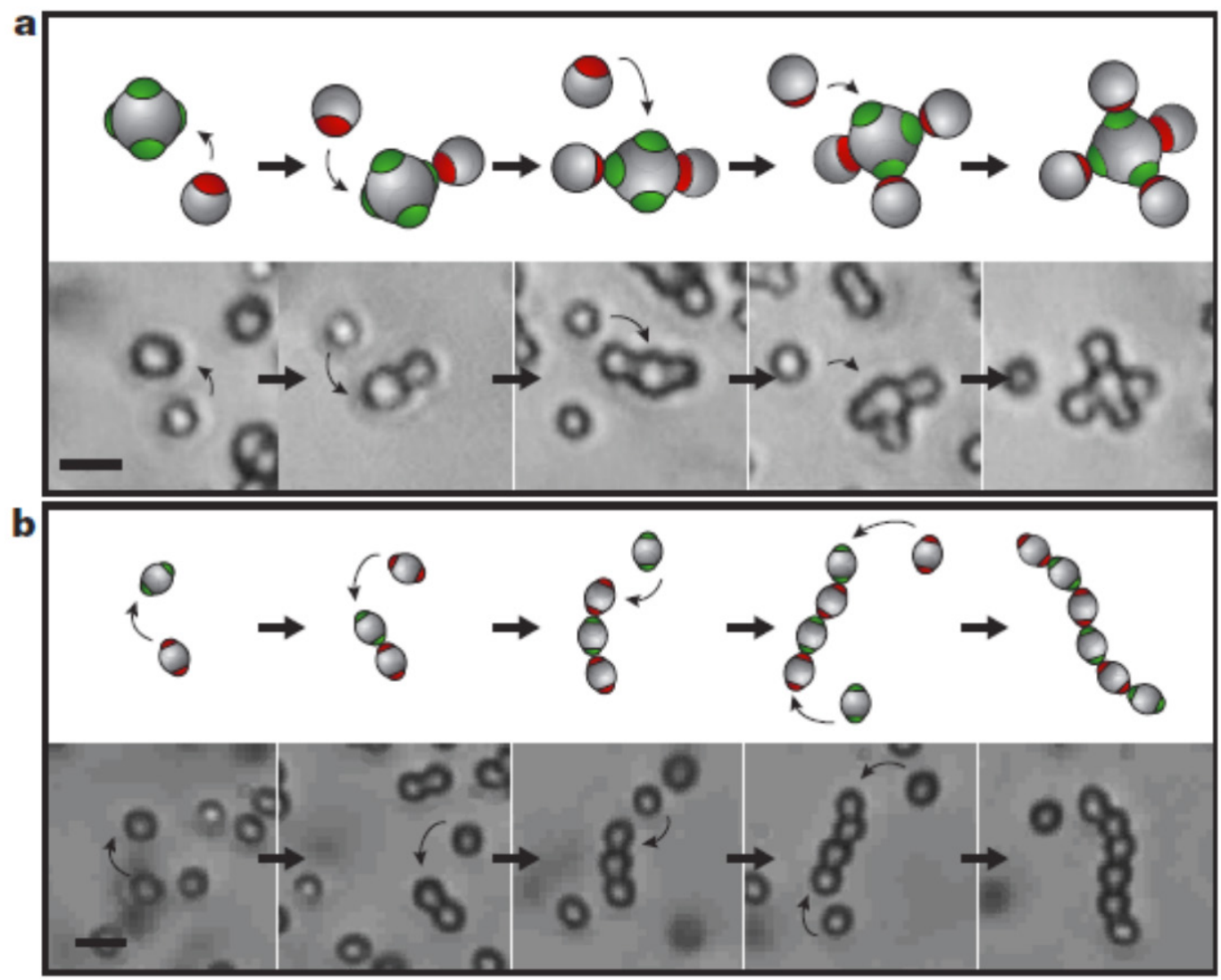

Figure 4 | Step-wise sequential kinetics of supracolloidal reactions. Schematic images and snapshots from movies show the step-by-step reactions between colloidal atoms. Bent arrows point from the colloidal atom to the site where it is going to attach. Straight arrows indicate time sequence. a, Monovalent particles attach to tetravalent particle, one by one, forming an $\mathrm{AB}_{4}$ type colloidal molecule. b, Complementary divalent particles polymerize into linear chain structure. Scale bar, $2 \mu \mathrm{m}$. Movies of these processes are available in the Supplementary Materials. 
An obvious and important difference between the molecular and colloidal domains is the size of the constituents. The much larger colloids exhibit much slower dynamics and reaction kinetics, and thus can be observed in situ under an optical microscope. As shown in Fig. 4a, the formation of an $\mathrm{AB}_{4}$ molecule proceeds by the central tetravalent particle picking up monovalent particles, one at a time, over a period of about 30 minutes (see Supplementary Movie 2). In the case of divalent particle chain formation, the "polymerization" also follows a step-growth mechanism. Figure $4 \mathrm{~b}$ illustrates how a polymer chain can be extended by adding divalent particles one by one at the end (see Supplementary Movie 3). Alternatively, two polymer chains can fuse into a longer chain.

We can understand the stepwise growth mechanism by examining the kinetics of formation of the $\mathrm{AB}_{3}$ molecules (see Supplementary Fig. S5, Movie 4). We first heat a trivalent and monovalent particle mixture, with monovalent particles in large excess, to $55^{\circ} \mathrm{C}$, well above the melting temperature $T_{m}\left(50^{\circ} \mathrm{C}\right)$ of the DNA, thus causing the particles to dissociate. The system is then quenched to room temperature, well below $T_{m}$, so that the reaction kinetics is controlled by diffusion and by the size of the sticky patches. The collision frequency between monovalent and trivalent particles can be estimated from the well-known Smoluchowski equation ${ }^{42}$ :

$$
J \approx 4 \pi\left(b_{m}+b_{t}\right)\left(D_{m}+D_{t}\right) C_{m}
$$

where $b_{m}=0.49 \mu \mathrm{m}$ is the radius of the monovalent particle, $b_{t}=0.91 \mu \mathrm{m}$ is the radius of the trivalent particle, and $C_{m}$ is the number concentration of the monovalent particles, estimated to be 1 particle per $50 \mu^{3}$, based on direct observation. The diffusion coefficients are about $D_{m}=0.50 \mu \mathrm{m}^{2} / \mathrm{s}$ and $D_{t}=0.28 \mu \mathrm{m}^{2} / \mathrm{s}$ for the monovalent and trivalent particles, respectively. These values give a collision frequency between trivalent and monovalent particles of $0.27 \mathrm{~s}^{-1}$ or a time between collisions of $3.6 \mathrm{~s}$. Not every collision results in a bond, however, due to the anisotropic nature of patchy particles. Only collisions between patches with complementary DNA can result in adhesion, with a rate proportional to the patch size. Thus, smaller patches form bonds at a rate slower than larger patches.

We define $A$ as the fractional surface area of a particle comprising patches (see Supplementary Fig. S4, S5), with values estimated from SEM measurements of $A_{m}=0.23$ for the monovalent particle and $A_{t}=0.077$ all three patches of the trivalent particle (see Supplementary Tab. 1). The estimated reaction time for the first 
monovalent particle to adhere to a trivalent particle is $1 / J A_{m} A_{t}$ or about $3.4 \mathrm{~min}$. The area $A$ for the trivalent-monovalent particle assembly intermediately falls to 0.040 because one of the 3 patches is covered, and because the attached monovalent particle increases the total surface area of the complex. With two monovalent particles attached, $A$ falls to 0.016. Thus the times for the second and third monovalent particles to attach are estimated to be $6.5 \mathrm{~min}$ and $16 \mathrm{~min}$, respectively, consistent with the times observed experimentally and with the observed stepwise assembly of patchy particles. (see Supplementary Movie 2).

\section{Discussion}

We expect that our colloids with valence can assemble not only into the molecular analogues shown here, but also into bulk colloidal phases of fundamental and practical interest. Tetrahedrally-coordinated glasses, diamond crystals, and empty liquids ${ }^{43}$ have all have proven difficult or impossible to make with existing colloids but should be accessible using our method. However, making scalable quantities of purified divalent and higher valence colloidal atoms remains a challenge, owing to the limitations of fractionation by density gradient centrifugation. This difficulty might be overcome by large-scale separations ${ }^{44}$, or developing methods that produce clusters with controlled morphology so that no separation is needed. Indeed, the swelling and functionalization methods we use to make our colloidal particles could readily be adapted for use with clusters made using other recently-developed techniques ${ }^{22,25,45}$.

The ability to design colloidal particles with a variety of well-controlled 3-dimensional bonding symmetries opens a wide spectrum of new structures for colloidal self-assembly, beyond colloidal assemblies whose structures are defined primarily by repulsive interactions and colloidal shape. Furthermore, the specificity of DNA interactions between patches means that colloids with different properties, such as size, colour, chemical functionality, or electrical conductivity could be linked in well-defined sequences and orientations to make new functional materials. Such materials might include photonic crystals with programmed distributions of defects or 3-dimensional electrically wired networks. Future work could exploit the ability to independently vary the valence and interaction strength, which in atomic systems are coupled by underlying quantum mechanical rules. Our patchy particles might therefore not only mimic atoms, but could function as "designer atoms" 46 whose reactions, unconstrained by the rules that govern bonding at the atomic scale, could yield structures that have no analogues in any atomic or molecular system. $^{43,47}$ 


\section{Methods Summary}

Cross-linked amidinated polystyrene microspheres were synthesized using a surfactant-free emulsion polymerization method. The amidinated clusters were prepared as described by Manoharan et al. Shearing conditions were optimized to control cluster distribution. A two-stage swelling and polymerization method was employed to encapsulate the clusters thereby fabricating mixtures of patchy particles that were separated by density gradient centrifugation. After purification, the separated particles were dispersed in $10 \mathrm{mM}$ PBS buffer solution (pH: 7.42, NaCl: $100 \mathrm{mM}$ ) containing $0.1 \%(\mathrm{w} / \mathrm{w})$ Triton X-100 and reacted with sulfo-NHS-biotin to convert the functionalities on the patches from amidines to biotins. 5'-Biotin-DNA was mixed with fluorescent streptavidin in a 1:1 ratio and the resulting complex was used to attach DNA to the biotinylated patches. Finally, the DNA-containing particles were washed with and dispersed in an aqueous PBS buffer solution containing 1\% (w/w) Pluronic F127. This suspension was used for all self-assembly experiments. For the self-assembly studies, the mixture of interest was sealed in a hydrophobic capillary tube and imaged using optical microscopy. Particles dried on a silicon wafer were imaged by field-emission scanning electron microscope. The fluorescent images were obtained using a Leica SP5 confocal fluorescence microscope. Laser lines at $488 \mathrm{~nm}$ and $633 \mathrm{~nm}$ were used to excite the green and red fluorescence.

Full Methods and any associated references are available in the online version of the paper at www.nature.com/nature.

\section{References}

1 Glotzer, S. C. \& Solomon, M. J. Anisotropy of building blocks and their assembly into complex structures. Nature Material 6, 557-562 (2007).

2 Sacanna, S. \& Pine, D. J. Shape-anisotropic colloids: Building blocks for complex assemblies. Current Opinion in Colloid \&amp; Interface Science 16, 96-105 (2011).

3 Rossi, L. et al. Cubic crystals from cubic colloids. Soft Matter 7, 4139-4142 (2011).

4 Meng, G., Arkus, N., Brenner, M. P. \& Manoharan, V. N. The Free-Energy Landscape of Clusters of Attractive Hard Spheres. Science 327, 560-563 (2010).

5 Manoharan, V. N., Elsesser, M. T. \& Pine, D. J. Dense Packing and Symmetry in Small Clusters of Microspheres. Science 301, 483-487 (2003).

6 Kraft, D. J. et al. Self-Assembly of Colloids with Liquid Protrusions. Journal of the American Chemical Society 131, 1182-1186 (2008). 

464, 575-578 (2010).

8 Zoldesi, C. I., van Walree, C. A. \& Imhof, A. Deformable Hollow Hybrid Silica/Siloxane Colloids by Emulsion Templating. Langmuir 22, 4343-4352 (2006).

Li, F., Josephson, D. P. \& Stein, A. Colloidal Assembly: The Road from Particles to Colloidal Molecules and Crystals. Angewandte Chemie International Edition 50, 360-388 (2011).

10 Leunissen, M. E. et al. Ionic colloidal crystals of oppositely charged particles. Nature 437, 235-240 (2005).

11 Macfarlane, R. J. et al. Nanoparticle Superlattice Engineering with DNA. Science 334, 204-208 (2011).

12 Velikov, K. P., Christova, C. G., Dullens, R. P. A. \& van Blaaderen, A. Layer-by-Layer Growth of Binary Colloidal Crystals. Science 296, 106-109 (2002).

13 Ho, K. M., Chan, C. T. \& Soukoulis, C. M. Existence of a photonic gap in periodic dielectric structures. Physical Review Letters 65, 3152-3155 (1990).

14 Nelson, D. R. Toward a Tetravalent Chemistry of Colloids. Nano Letters 2, 1125-1129 (2002).

15 Glotzer, S. C. Some Assembly Required. Science 306, 419-420 (2004).

16 Manoharan, V. N. Building Materials with Colloidal Spheres Ph. D thesis, University of California, Santa Barbara, (2004).

Breed, D. R. Engineered Colloids: Patchy Particles with Reversible, Directional Interactions Ph. D thesis, University of California, Santa Barbara, (2007). Bianchi, E., Blaak, R. \& Likos, C. N. Patchy colloids: state of the art and perspectives. Physical Chemistry Chemical Physics 13, 6397-6410 (2011).

Zhang, Z. \& Glotzer, S. C. Self-Assembly of Patchy Particles. Nano Letters 4, 1407-1413 (2004).

Xu, X., Rosi, N. L., Wang, Y., Huo, F. \& Mirkin, C. A. Asymmetric Functionalization of Gold Nanoparticles with Oligonucleotides. Journal of the American Chemical Society 128, 9286-9287 (2006).

21 Huo, F., Lytton-Jean, A. K. R. \& Mirkin, C. A. Asymmetric Functionalization of Nanoparticles Based on Thermally Addressable DNA Interconnects. Advanced Materials 18, 2304-2306 (2006). Hong, L., Cacciuto, A., Luijten, E. \& Granick, S. Clusters of Amphiphilic Colloidal Spheres. Langmuir 24, 621-625 (2008). Chen, Q., Bae, S. C. \& Granick, S. Directed self-assembly of a colloidal kagome lattice. Nature 469, 381-384 (2011). Nanoparticle Shape Anisotropy Dictates the Collective Behavior of Surface-Bound Ligands. Journal of the American Chemical Society 133, 18865-18869 (2011). 
Kraft, D. J., Groenewold, J. \& Kegel, W. K. Colloidal molecules with well-controlled bond angles. Soft Matter 5, 3823-3826 (2009).

Cho, Y.-S. et al. Particles with Coordinated Patches or Windows from Oil-in-Water Emulsions. Chemistry of Materials 19, 3183-3193 (2007).

Zhang, G., Wang, D. \& Möhwald, H. Patterning Microsphere Surfaces by Templating Colloidal Crystals. Nano Letters 5, 143-146 (2005).

Pawar, A. B. \& Kretzschmar, I. Multifunctional Patchy Particles by Glancing Angle Deposition. Langmuir 25, 9057-9063 (2009).

Pawar, A. B. \& Kretzschmar, I. Fabrication, Assembly, and Application of Patchy Particles. Macromolecular Rapid Communications 31, 150-168 (2010).

Ottewill, R. \& Shaw, J. Studies on the preparation and characterization of monodisperse polystyrene latices. Colloid \& Polymer Science 215, 161-166 (1967).

Ugelstad, J., Kaggerud, K. H., Hansen, F. K. \& Berge, A. Absorption of low molecular weight compounds in aqueous dispersions of polymer-oligomer particles, 2: A two step swelling process of polymer particles giving an enormous increase in absorption capacity. Makromol. Chem. 180, 737-744 (1979). composite, monosized polymer particles. Die Makromolekulare Chemie 10, 215-234 (1985).

33 Walther, A. \& Muller, A. H. E. Janus particles. Soft Matter 4, 663-668 (2008).

34 Zerrouki, D. et al. Preparation of Doublet, Triangular, and Tetrahedral Colloidal Clusters by Controlled Emulsification. Langmuir 22, 57-62 (2005).

Mirkin, C. A., Letsinger, R. L., Mucic, R. C. \& Storhoff, J. J. A DNA-based method for rationally assembling nanoparticles into macroscopic materials. Nature 382, 607-609 (1996).

Alivisatos, A. P. et al. Organization of 'nanocrystal molecules' using DNA. Nature 382, 609-611 (1996).

Leunissen, M. E. et al. Switchable self-protected attractions in DNA-functionalized colloids. Nat Mater 8, 590-595 (2009).

Nykypanchuk, D., Maye, M. M., van der Lelie, D. \& Gang, O. DNA-guided crystallization of colloidal nanoparticles. Nature 451, 549-552 (2008).

Zhang, Z., Keys, A. S., Chen, T. \& Glotzer, S. C. Self-Assembly of Patchy Particles into Diamond Structures through Molecular Mimicry. Langmuir 21, 11547-11551 (2005).

Chen, Q. et al. Supracolloidal Reaction Kinetics of Janus Spheres. Science 331, 199-202 (2011).

41 Dreyfus, R. et al. Aggregation-disaggregation transition of DNA-coated colloids: Experiments and theory. Physical Review E 81, 041404 (2010). 

silico. The Journal of Chemical Physics 134, 174502-174508 (2011). Dumbbells. Langmuir 21, 11510-11517 (2005). Chemie International Edition 48, 361-365 (2009).

46 Frenkel, D. Playing Tricks with Designer "Atoms". Science 296, $65-66$ (2002).

47 Miller, M. A. \& Wales, D. J. Novel Structural Motifs in Clusters of Dipolar Spheres: Knots, Links, and Coils. The Journal of Physical Chemistry B 109, 23109-23112 (2005).

Acknowledgments We thank M. T. Elsesser, D. Kraft, and G.-R. Yi for helpful discussions. This work was supported partially by the MRSEC Program of the National Science Foundation under Award Number DMR-0820341. Additional financial support was provided by the National Science Foundation (ChE-0911460). We acknowledge support from the MRI program of the National Science Foundation under Award DMR-0923251 for the purchase of a Zeiss field emission scanning electron microscope.

Author Contributions V.N.M. and D.J.P. conceived of patchy particles and how to make them. D.R.B developed the functionalization of patches. Yf.W. and Y.W. optimized the control of patch size and DNA functionalization, performed the experiments, and collected and analyzed the data. A.D.H. helped with particle synthesis. L.F. designed the DNA sequences and modeled the kinetics. M.W. and D.J.P. supervised the project. D.J.P., Yf.W., and Y.W. wrote the paper with revisions from all of the authors.

Author Information Reprints and permissions information is available at www.nature.com/reprints. The authors declare no competing financial interests. Readers are welcome to comment on the online version of this article at www.nature.com/nature. Correspondence and requests for materials should be addressed to D.J.P. (pine@nyu.edu) or M.W. (marcus.weck@nyu.edu). 


\section{Methods}

\section{Microspheres and clusters formation}

Amidinated poly(styrene) microspheres were synthesized using the standard surfactant-free emulsion polymerization method described in the literature ${ }^{30}$ (monomer: styrene, initiator: 2,2'-azobis(isobutyramidine) dihydrochloride (AIBA), cross linker: $3 \mathrm{~mol} \%$ divinyl benzene). The amidinated microsphere clusters were prepared as described by Manoharan et al. ${ }^{5}$ In a variation to the original report, we used sodium dodecyl sulfate (SDS) as surfactant and changed the shear rate to control cluster distribution. The following two shear experiments were carried out as described in the main document and corresponding to patchy particle distributions in Figure S1: condition a (low shear): 90 seconds at $8000 \mathrm{rpm}, 60$ seconds at $9500 \mathrm{rpm}$, and then 60 seconds at 13500 rpm; condition b (high shear): 90 seconds at 8000 rpm, 90 seconds at 9500 rpm, and the 120 seconds at $13500 \mathrm{rpm}$. The final clusters were washed with an aqueous solution of $0.1 \%$ SDS followed by repeated centrifugation and redispersion. Finally, we adjust the clusters concentration to $1 \%$, w/w and modified the $\mathrm{pH}$ to 2.93 using $\mathrm{HCl}$.

\section{Patchy particle fabrication}

A two-stage swelling and polymerization method was employed to encapsulate the clusters to fabricate patchy particles. Typically, $10 \mathrm{~mL}$ of the cluster suspension was charged into a $50 \mathrm{~mL}$ 2-neck flask along with a magnetic stir bar. The flask was submerged in an oil bath and the temperature was set to $35^{\circ} \mathrm{C}$. 1 $\mathrm{mL}$ of acetone was added and the suspension was stirred at $300 \mathrm{rpm}$. In a separated glass vial, $50 \mathrm{mg}$ of benzoyl peroxide were dissolved in $0.63 \mathrm{~mL}$ of 1,2-dichloroethane. Then, $0.88 \mathrm{~mL}$ of 1 -chlorodecane was added to the vial followed by the addition of $5 \mathrm{~mL}$ of an aqueous solution of $0.1 \%$ SDS. The resulting mixture was then vortexed to create an emulsion, from which $200 \mu \mathrm{L}$ were added to the cluster suspension. The resulting mixture was stirred for 12 hours at $35^{\circ} \mathrm{C}$. Then, the acetone was removed via evaporation under reduced pressure (30 mmHg). The flask was equipped with a condenser containing an oil bubbler at the top. Using a needle, nitrogen was bubbled through the suspension for 30 minutes. Then, $1 \mathrm{~mL}$ of styrene (with inhibitor removed) was added and allowed to swell the clusters. After 2 hours, the temperature was raised to $65^{\circ} \mathrm{C}$ to initiate polymerization. The polymerization was allowed to take place for 14 hours. Then, the reaction was cooled to room temperature, which terminates the polymerization yielding the desired patchy particles as a mixture. 


\section{Density gradient centrifugation}

The patchy particle mixture was separated by density gradient centrifugation. A $5 \%-20 \% \mathrm{w} / \mathrm{w}$ linear gradient of glycerol in water solution was made by a "two-jar" type gradient maker. Typically, $300 \mu \mathrm{L}$ of the patchy particle mixture was loaded on top of $12 \mathrm{~mL}$ of the gradient solution followed by centrifugation for 24 minutes at $4000 \mathrm{rpm}$ at $20^{\circ} \mathrm{C}$. Individual bands were extracted carefully using a syringe with pipetting needles. The individual fractions were washed first with an aqueous solution containing $0.1 \% \mathrm{w} / \mathrm{w}$ Triton $\mathrm{X}-100$ (3 times) followed by an aqueous solution containing $10 \mathrm{mM}$ PBS buffer (pH: 7.42, NaCl: $100 \mathrm{mM}$ ) and $0.1 \% \mathrm{w} / \mathrm{w}$ Triton X-100 (3 times).

To obtain the quantity of particles in each band we used the mass of poly(styrene) and the size of the particles. For this, the particles were washed with deionized water, dried under vacuum, and weighed. For higher valence particles, the quantity was estimated by measuring the number ratio relative to lower valence particles under a microscope.

\section{Biotin functionalization}

$1 \mathrm{mg}$ of sulfo-NHS-biotin was charged into a dram vial containing a stir bar. 0.5 $\mathrm{mL}$ of patchy particles of interest was added to the vial and the suspension was allowed to stir for 12 hours. Biotin was used in large excess. Unreacted biotin was removed by washing the functionalized particles six times with an aqueous solution containing $10 \mathrm{mM}$ PBS buffer (pH: 7.42, NaCl: $100 \mathrm{mM}$ ) and 0.1\% w/w Triton X-100.

\section{DNA conjugation}

The single stranded oligonucleotides used in this study were purchased from Integrated DNA Technologies USA. The sequences and functional groups are shown below:

G: $5^{\prime}$ - /5BioTEG/AT CGC TAC CCT TCG CAC AGT CAA TCC AGA GAG CCC TGC CTT TCA TTA CGA CCT ACT TCT AC - 3'.

R: 5' - /5BioTEG/AT CGC TAC CCT TCG CAC AGT CAA TCC AGA GAG CCC TGC CTT TCA TTA CGA GTA GAA GTA GG - 3'.

Palindrome (P): 5' - /5BioTEG/AT CGC TAC CCT TCG CAC AGT CAA TCC AGA GAG CCC TGC CTT TCA TTA CGA TAC GCG TA - 3'.

Complementary Strand for the backbone (CS): 5' - CGT AAT GAA AGG CAG GGC TCT CTG GAT TGA CTG TGC GAA GGG TAG CGA T - 3'. 
The 5'-biotin-DNA was prepared by mixing G, $\mathrm{R}$, or $\mathrm{P}$ with CS in a 1:1.5 ratio, heating it to $95^{\circ} \mathrm{C}$, and then cooling it slowly over a two hour period to $25^{\circ} \mathrm{C}$.

The 5'-biotin-DNA was mixed with streptavidin (Life tech., $2 \mathrm{mg} / \mathrm{mL}$, green or red fluorescent labeled) in 1:1 molar ratio in a centrifuge tube and agitated for 1 hour. The resulting DNA-streptavidin complex was then attached to the biotin patchy particles. Typically, we added a $100 \mu \mathrm{L}$ suspension of biotin patchy particles to $10 \mu \mathrm{L}$ of the DNA-streptavidin complex and agitated the mixture for 3 hours at $25^{\circ} \mathrm{C}$. The resulting particles were washed with and dispersed in an aqueous solution of PBS buffer solution containing 1\% w/w Pluronic F127 as surfactant. This dispersion can be stored at $4{ }^{\circ} \mathrm{C}$ and directly used for the self-assembly studies.

\section{Self-assembly}

For the self-assembly studies, the patchy particles of interest were combined and the mixture transferred to a flat capillary tube $(2 \mathrm{~mm} \times 100 \mu \mathrm{m} \times 1 \mathrm{~cm})$. The capillary tube was pretreated with plasma and exposed toHexamethyldisilazane vapor to make it hydrophobic. After addition of the sample, the capillary tube was sealed by UV glue or wax. The capillary tube temperature was controlled by using a Linkam microscope hot stage.

\section{Microscopy}

Particles (clusters or patchy particles) in the dried state were imaged using a MERLIN (Carl Zeiss) field emission scanning electron microscope (FE-SEM). The samples were prepared by placing a drop of a dilute aqueous particle suspension on a silicon wafer followed by evaporation of the water under vacuum.

Fluorescent images were taken using a Leica SP5 confocal fluorescence microscope. Laser lines $488 \mathrm{~nm}$ and $633 \mathrm{~nm}$ were used to excite green and red fluorescence.

Some of the microscope images were digitally post-processed to improve brightness and/or contrast. 


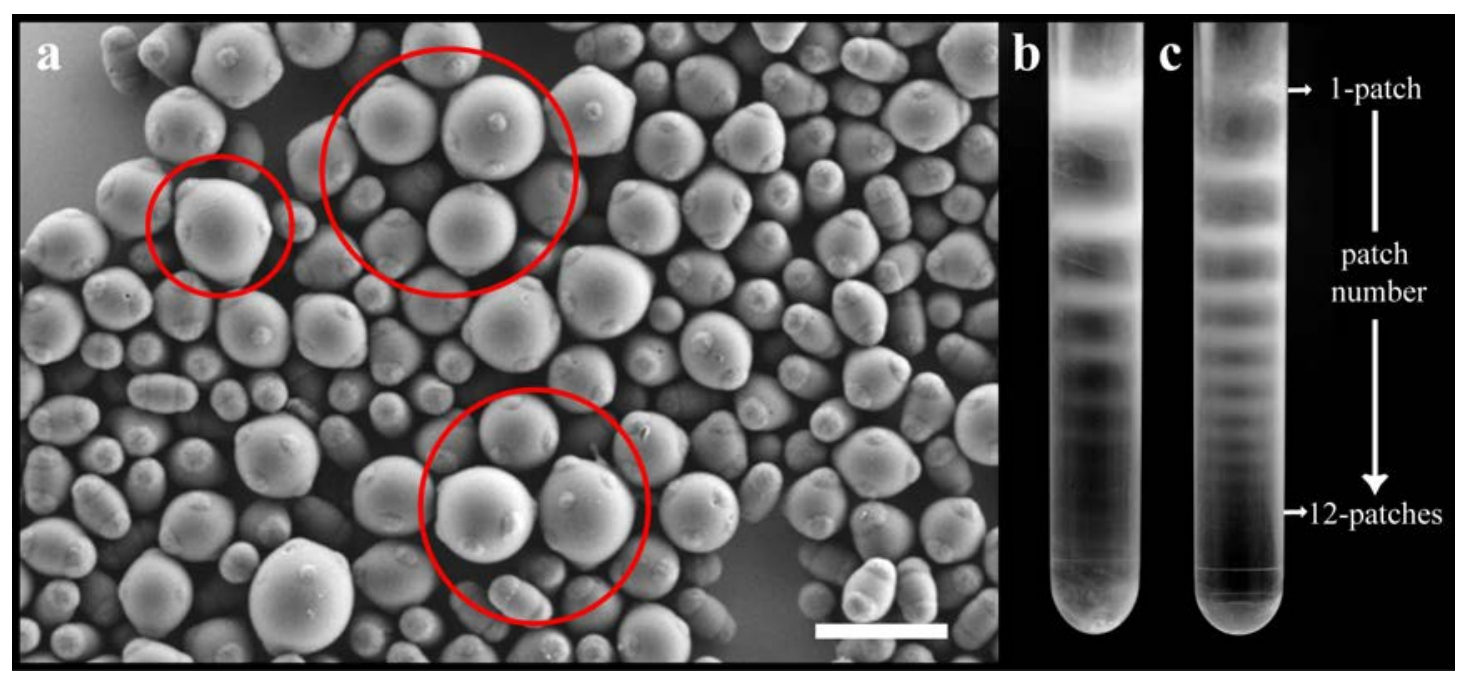

Figure S1 | Patchy particle distribution. a, Electron micrograph showing a mixture of amidinated patchy particles before they are separated by density gradient centrifugation. Particles of the same order $n$ have the same configuration. Some of the higher order $(n>7)$ particles are circled. Their patch geometries extend beyond those of atomic orbitals. Scale bar, $2 \mu \mathrm{m}$. $\mathbf{b}$ and $\mathbf{c}$, Optical micrographs of test tubes containing patchy particle suspensions fractionated by density gradient centrifugation. Each white band represents a region of high concentration of identical patchy particles. $\quad \mathbf{b}$, Patchy particles are fabricated from clusters using high shear, resulting in the formation of relatively more lower valence particles. Eight bands are visible with the highest number being the monovalent patchy particles. c, Patchy particles are fabricated from clusters using low shear. This results in the formation of a greater percentage of patchy particles with higher valence. Twelve distinct bands corresponding to patchy particles, from monovalent to 12-valent, are visible, with the most pronounced band corresponding to trivalent patchy particles. 


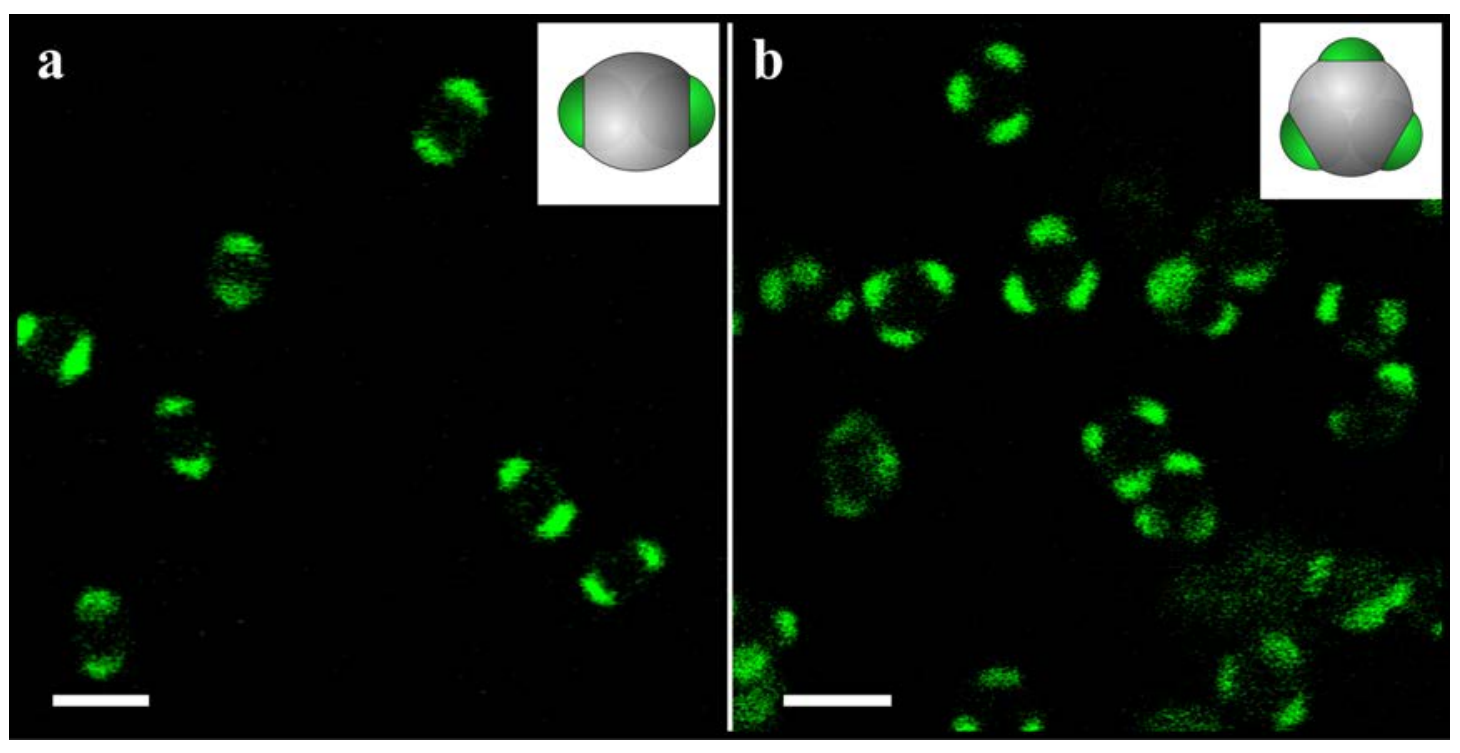

Figure S2 | Confocal fluorescent images of purified DNA patchy particles. Patchy particles are separated and functionalized with DNA using a biotinstreptavidin-biotin linkage. For visualization, the fluorescently-labeled streptavidin was used. a, Purified divalent (2-patch) and b, trivalent (3-patch) particles with green fluorescence only on the patches. Scale bar, $2 \mu \mathrm{m}$. 


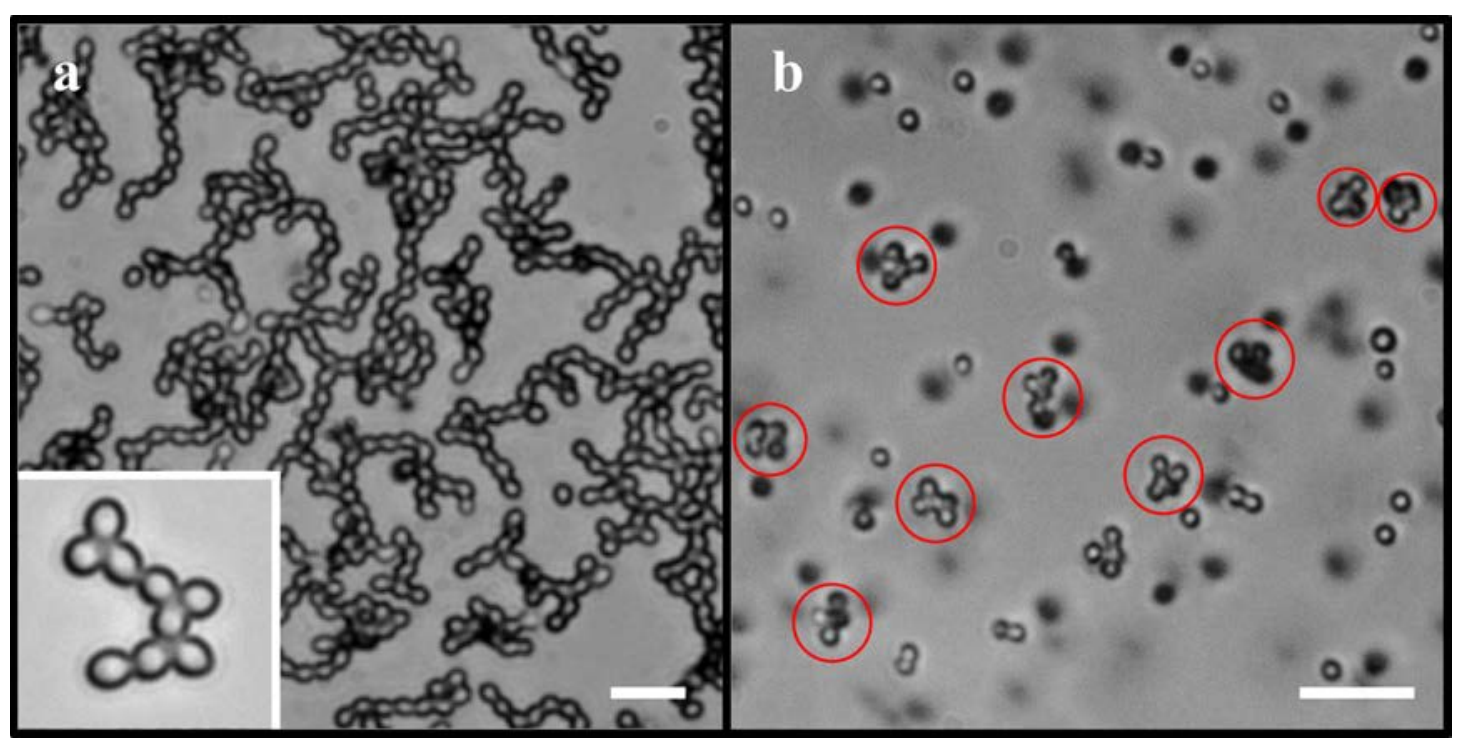

Figure S3 | Microscopic images of assembled structures. a, Polymer network formed via the self-assembly of divalent particles with relatively large patches (particles from Figure S3c). The inset shows a higher magnification of a branched polymer. b, $\mathrm{AB}_{4}$ colloidal molecules (circled) assembled using a five folds excess of the monovalent particles. Scale bar, $10 \mu \mathrm{m}$. 


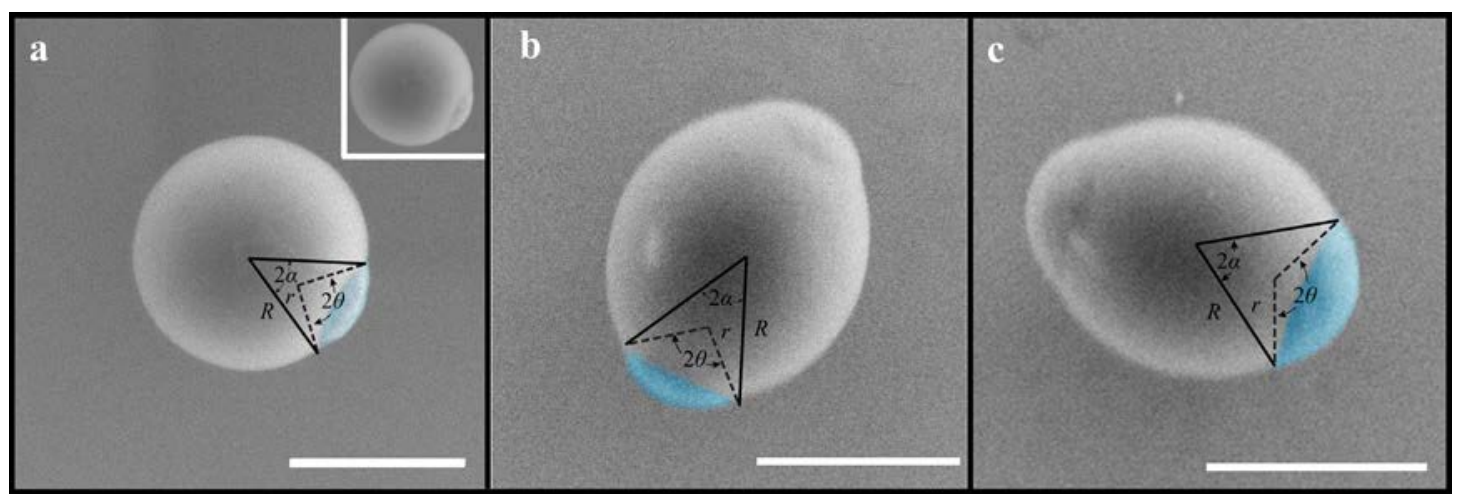

Figure S4 | Patch size and its effect on assembled structures. a, Electron micrograph of a monovalent particle with a small patch in blue false-color to enhance its visibility (inset shows particle without false-color). To study the effect of patch size on particle assembly, we define the fraction $A$ of the particle surface area covered by the patches. We deduce $A$ from measurements of $R, r, \alpha$ and $\theta$ using Eq. S1, where $R$ is the distance from the patchy particle center to the patch edge and $r$ is the radius of the primary microspheres, as shown above. The angles $2 \alpha$ and $2 \theta$ are defined above while $n$ is the number of patches per particle. The values of $r / R, \alpha, \theta$, and $A$ are listed in Supplementary Table 1 . For $\theta<30^{\circ}$, it is geometrically impossible for more than one particle to bind to a patch of an identical patchy particle. When $\theta>30^{\circ}$, the probability of two particles binding to a single patch is still small because the matrix also provides steric hindrance (depending on $\alpha$ ). In our experiments, we observe two particles binding to a single patch only for $\alpha>30^{\circ}$ and $\theta>60^{\circ}$. b, Electron micrograph of a divalent particle with small patches. This batch of divalent particles was used for building linear $\mathrm{AB}_{2}$ colloidal molecules and polymers/oligomers. c, A divalent particle with relatively large patches. Divalent particles from this batch were used for the "cis" and "trans" $\mathrm{AB}_{2}$ colloidal molecule assemblies and the formation of branched polymers. Scale bars, $1 \mu \mathrm{m}$. 


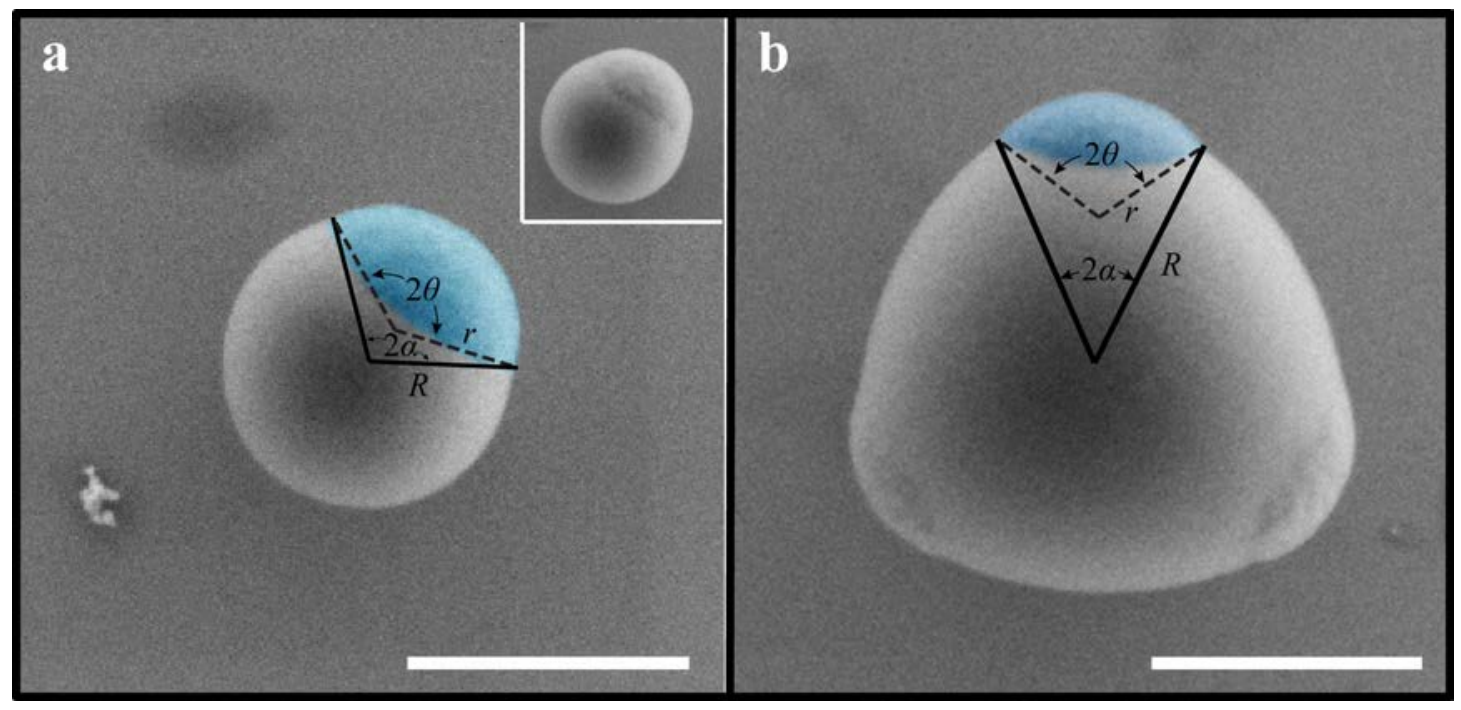

Figure S5 | Patchy particles used to study the colloidal molecule formation kinetics. Electron micrograph of (a) a monovalent particle with $A=0.23$ and (b) a trivalent particle $(A=0.077)$. This combination was chosen because our calculations indicated that the self-assembly between these two particles should occur in a few minutes to a half hour. If particles with much smaller patches were to be used, the assembly times would increase significantly. Using monovalent particles with large patches facilitates faster binding but may allow multiple trivalent particles to bind to a single monovalent particle. To prevent two trivalent particles from binding to a single monovalent particle, the concentration (number of equivalents) of monovalent particles was increased, thereby suppressing the binding of two trivalent particles to a single monovalent particle. Indeed, we observed fast self-assembly with directionality maintained. Scale bar, $1 \mu \mathrm{m}$. 


\section{Supplementary Equation 1:}

The fraction $A$ of the area occupied by patches on a patchy particle is given by

$$
A=\frac{n(1-\cos \theta)}{[2-n(1-\cos \alpha)]\left(R^{2} / \mathrm{r}^{2}\right)+n(1-\cos \theta)}
$$

where $n$ is the number of patches on the particles and $R, r, \alpha$ and $\theta$ are defined in Fig. S3. 
Supplementary Table 1:

\begin{tabular}{ccccc}
\hline Figure & $\boldsymbol{r} / \boldsymbol{R}$ & $\boldsymbol{\theta}$ & $\boldsymbol{\alpha}$ & $\boldsymbol{A} \%$ \\
\hline 3a & 0.56 & $45^{\circ}$ & $26^{\circ}$ & 4.6 \\
3b & 0.56 & $54^{\circ}$ & $26^{\circ}$ & 13 \\
3c & 0.60 & $69^{\circ}$ & $34^{\circ}$ & 22 \\
4a & 0.87 & $68^{\circ}$ & $53^{\circ}$ & 23 \\
4b & 0.48 & $38^{\circ}$ & $24^{\circ}$ & 7.7 \\
\hline
\end{tabular}




\section{Supplementary Movie Notes and Legends:}

All movies were taken at $25^{\circ} \mathrm{C}$. Particles were suspended in an aqueous PBS buffer solution ( $\mathrm{pH}=7.4)$ containing surfactant (1\% w/w Pluronic F127). In Movie 1, the system had been stabilized for a few hours at $25^{\circ} \mathrm{C}$. Movies 2, 3, and 4 , were taken immediately after a rapid quench from $55^{\circ} \mathrm{C}$.

Supplementary Movie 1: A collection of $\mathrm{AB}_{\mathrm{n}}(\mathrm{n}=1-4)$ colloidal molecules assembled from monovalent, divalent, trivalent or tetravalent particles at the central position. The movie is acquired and displayed at real time. Scale bar, 2 $\mu \mathrm{m}$.

Supplementary Movie 2: Kinetics of $\mathrm{AB}_{4}$ colloidal molecule formation. Starting from a bare tetravalent particle, the kinetics is followed as four monovalent particles are assembled onto the tetravalent particle successively. The time intervals between each set of clips are indicated below the movies. The movie is acquired at $24 \mathrm{fr} / \mathrm{s}$ and played at a rate shown in the upper left corner. Scale bar, $3 \mu \mathrm{m}$.

Supplementary Movie 3: Polymerization kinetics of the assembly of complementary divalent particles. A linear polymer chain starts from a divalent particle, which picks up other single divalent particles sequentially. The movie is acquired at $10 \mathrm{fr} / \mathrm{s}$ and played at a rate indicated in the lower left corner. Scale bar, $2 \mu \mathrm{m}$.

Supplementary Movie 4: Kinetics of $\mathrm{AB}_{3}$ colloidal molecule formation. Trivalent particles act as central atoms, picking up monovalent particles one at a time. Most trivalent particles assemble a monovalent particle before the first trivalent particles pick up a second monovalent one. The same is true for the next assembly step. Only after most trivalent particles have acquired two monovalent particles do third particles begin to attach. The movie is acquired at $24 \mathrm{fr} / \mathrm{s}$ and played at a rate indicated in the lower left corner. Scale bar, $3 \mu \mathrm{m}$. 